\title{
The equivalence between singular point quantities and Liapunov constants on center manifold
}

\author{
Qinlong Wang ${ }^{1^{*}}$ and Wentao Huang ${ }^{2}$
}

\author{
* Correspondence: wqinlong@163. \\ com \\ 'School of Information and \\ Mathematics, Yangtze University, \\ Jingzhou 434023, People's Republic \\ of China \\ Full list of author information is \\ available at the end of the article
}

\begin{abstract}
The algorithm of singular point quantities for an equilibrium of three-dimensional dynamics system is studied. The explicit algebraic equivalent relation between singular point quantities and Liapunov constants on center manifold is rigorously proved. As an example, the calculation of singular point quantities of the Lü system is applied to illustrate the advantage in investigating Hopf bifurcation of threedimensional system.
\end{abstract}

MR (2000) Subject Classification: 34C23, 34C28, 37GXX.

Keywords: three-dimensional system, multiple Hopf bifurcation, singular point quantities, center manifold

\section{Introduction}

We consider the real three-dimensional differential autonomous systems which take the form

$$
\dot{\mathbf{x}}=A \mathbf{x}+\mathbf{f}(\mathbf{x})
$$

where $\mathbf{x} \in \mathbb{R}^{3}, A \in \mathbb{R}^{3 \times 3}, \mathbf{f}(\mathbf{x}) \in C^{2}$ with $\mathbf{f}(\mathbf{0})=\mathbf{0}, \mathbf{D f}(\mathbf{0})=\mathbf{0}$, then the origin is an equilibrium. The systems (1) usually involve many important nonlinear dynamical models such as Lotka-Volterra system [1,2] and Lorenz system [3,4].

As far as Hopf bifurcation of the origin of systems (1) is concerned, the Jacobian matrix $A$ at the origin should have a pair of purely imaginary eigenvalues and a negative one. In general, one can apply firstly the center manifold theorem to reduce the system (1) to a two-dimensional system [5], then compute Liapunov constants or the bifurcation formulae based on Liapunov functions-Poincaré theory [6,7]. However, this traditionary way has quite complicated course of determining coefficients of the twodimensional reduced equations, and for bifurcation formulae or Liapunov coefficient $[6,8]$, usually, only the first value is obtained, thus just one single limit cycle in the vicinity of the origin can be found.

Recently, the authors Wang et al. [9] introduced an algorithm of computing the singular point quantities on center manifold, which misses the above tedious course. In contrast to the usual ones, this algorithm is more convenient to investigate the multiple Hopf bifurcation at equilibrium of a three-dimensional system. However, it is possibly difficult to be approbated. For this reason, this paper will give the explicit relation between the singular point quantities and Liapunov constants of the origin of system (1).

(c) 2012 Wang and Huang; licensee Springer. This is an Open Access article distributed under the terms of the Creative Commons Attribution License (http://creativecommons.org/licenses/by/2.0), which permits unrestricted use, distribution, and reproduction in any medium, provided the original work is properly cited. 
And more we hope that the results presented here will stimulate the analysis of topological structure and dynamical behavior for a higher-dimensional system.

This paper is organized as follows. In Section 2, we give some preliminaries about Liapunov constants, the focal values and singular point quantities on center manifold for a three-dimensional system (1). In Section 3, the relation between the singular point quantities and Liapunov constants is investigated, and their algebraic equivalence is proved rigorously. In Section 4, the singular point quantities of the Lü system as an example are computed, then the existence of four limit cycles for this system is judged.

\section{The related definitions}

\subsection{Liapunov constants on center manifold}

We give firstly the definition of Liapunov constants for a three-dimensional system. Considering the Jacobian matrix $A$ at the origin of system (1) has a pair of purely imaginary eigenvalues and a negative one, then the system (1) can be put in the following form:

$$
\left\{\begin{array}{l}
\dot{x}_{1}=\ell_{1} x_{1}+\ell_{2} x_{2}+\sum_{k=2}^{\infty} X_{k}\left(x_{1}, x_{2}, x_{3}\right), \\
\dot{x}_{2}=\ell x_{1}-\ell_{1} x_{2}+\sum_{k=2}^{\infty} Y_{k}\left(x_{1}, x_{2}, x_{3}\right), \\
\dot{x}_{3}=-d x_{3}+\sum_{k=2}^{\infty} U_{k}\left(x_{1}, x_{2}, x_{3}\right)
\end{array}\right.
$$

Where $\dot{x}_{i}=\frac{\mathrm{d} x_{i}}{\mathrm{~d} \tau}(i=1,2,3)$, and $d, \lambda, \lambda_{1}, \ell_{2} \in \mathbb{R}\left(d>0, \ell_{1}^{2}+\ell \ell_{2}<0\right)$, and $X_{k}, Y_{k}, U_{k}$ are homogeneous polynomials in $x_{1}, x_{2}, x_{3}$ of degree $k$.

According to the center manifold theorem [5], the three-dimensional system (2) has an approximation to the center manifold taking the form

$$
u=u\left(x_{1}, x_{2}\right)=u_{2}\left(x_{1}, x_{2}\right)+\text { h.o.t. }
$$

where $u_{2}$ is a quadratic homogeneous polynomial in $x_{1}$ and $x_{2}$, and h.o.t. denotes the terms with orders greater than or equal to 3 . Substituting $u=u\left(x_{1}, x_{2}\right)$ into the equations of system (2), we can obtain a generic two-dimensional differential system with center-focus type linear part as follows

$$
\left\{\begin{array}{l}
\dot{x}_{1}=\ell_{1} x_{1}+\ell_{2} x_{2}+\sum_{k=2}^{\infty} \tilde{X}_{k}\left(x_{1}, x_{2}\right) \\
\dot{x}_{2}=\ell x_{1}-\ell_{1} x_{2}+\sum_{k=2}^{\infty} \tilde{Y}_{k}\left(x_{1}, x_{2}\right)
\end{array}\right.
$$

where $\tilde{X}_{k}, \tilde{Y}_{k}$ are homogeneous polynomials in $x_{1}, x_{2}$ of degree $k$, and their coefficients are polynomial functions of coefficients of the system (2). System (4) is often called the reduction system of (2). Correspondingly, one can take a generic Liapunov function

$$
H\left(x_{1}, x_{2}\right)=\kappa\left(\ell x_{1}^{2}-2 \ell_{1} x_{1} x_{2}-\ell_{2} x_{2}^{2}\right)+\sum_{k=3}^{\infty} H_{k}\left(x_{1}, x_{2}\right)
$$

where $\kappa$ can be an arbitrary non-zero real number, $H_{k}$ is a homogeneous polynomial in $x_{1}, x_{2}$ of degree $k$ and the coefficients of $H$ should satisfy 


$$
\left.\frac{\mathrm{d} H}{\mathrm{~d} \tau}\right|_{(4)}=V_{2} \Phi^{2}+V_{4} \Phi^{3}+\cdots+V_{2 m} \Phi^{m+1}+\cdots
$$

where $\Phi\left(x_{1}, x_{2}\right)=\left(x_{1}^{2}+x_{2}^{2}\right)$ or $x_{1}^{2}$ or $x_{2}^{2}$ or $\left(x_{1}+x_{2}\right)^{2}$ or other suitable forms [7,10,11].

Definition 2.1. $V_{2 m}$ in (6) is called the $m$ th Liapunov constant of the origin for system (2) or (4), $m=1,2, \ldots$.

\subsection{The focal values on center manifold}

In this part, we give the definition of the focal values for a three-dimensional system. One transformation matrix $P$ can be found such that the coefficient matrix $A$ of linear part of system (2) becomes the matrix $B$ as follows

$$
P^{-1} A P=P^{-1}\left(\begin{array}{lll}
\ell_{1} & \ell_{2} & 0 \\
\ell & -\ell_{1} & 0 \\
0 & 0 & -d
\end{array}\right) P=\left(\begin{array}{lll}
0 & -\omega & 0 \\
\omega & 0 & 0 \\
0 & 0 & -d
\end{array}\right) \triangleq B
$$

where $|P| \neq 0$ and $\omega=\left(-\ell_{1}^{2}-\ell \ell_{2}\right)^{1 / 2}>0$. Thus by a nondegenerate transformation $\left(x_{1}, x_{2}, x_{3}\right)^{\prime}=P(x, y, u)^{\prime}$, and after a time scaling: $t=\omega \tau$, the system (2) can be changed into the following system

$$
\left\{\begin{array}{l}
\frac{\mathrm{d} x}{\mathrm{~d} t}=-y+\sum_{k+j+l=2}^{\infty} A_{k j} x^{k} y^{j} u^{l}=X(x, y, u), \\
\frac{\mathrm{d} y}{\mathrm{~d} t}=x+\sum_{k+j+l=2}^{\infty} B_{k j l} x^{k} y^{j} u^{l}=Y(x, y, u), \\
\frac{\mathrm{d} u}{\mathrm{~d} t}=-d_{0} u+\sum_{k+j+l=2}^{\infty} d_{k j l} x^{k} y^{j} u^{l}=U(x, y, u)
\end{array}\right.
$$

where $x, y, u, A_{k j l}, B_{k j l}, d_{k j l} \in \mathbb{R}(k, j, l \in \mathbb{N})$ and $d_{0}=d / \omega$. Similarly, according to the center manifold theorem, putting certain approximation with the same form as (3) into (8), we get the following real planar polynomial differential system

$$
\left\{\begin{array}{l}
\frac{\mathrm{d} x}{\mathrm{~d} t}=-y+\sum_{k+j=2}^{\infty} A_{k j} x^{k} y^{j}=X(x, y) \\
\frac{\mathrm{d} y}{\mathrm{~d} t}=x+\sum_{k+j=2}^{\infty} B_{k j} x^{k} y^{j}=Y(x, y)
\end{array}\right.
$$

where $A_{k j}, B_{k j} \in \mathbb{R}(k, j \in \mathbb{N})$ and all $A_{k j}, B_{k j}$ are polynomial functions of coefficients of the system (8) or (2). System (9) is also called the equations on the center manifold or reduction system of (8). It is well-known, the origin of system (9) is center-focus type, and some significant work about it has been done in [12-14].

In order to define the focal values, we transform system (9) into the following form under the polar coordinates: $x=r \cos \theta, y=r \sin \theta$,

$$
\frac{\mathrm{d} r}{\mathrm{~d} t}=r \sum_{k=1}^{\infty} \varphi_{k}(\theta) r^{k}, \quad \frac{\mathrm{d} \theta}{\mathrm{d} t}=1+\sum_{k=1}^{\infty} \psi_{k}(\theta) r^{k}
$$

where $\phi_{k}(\theta)$ and $\psi_{k}(\theta), k=1,2,3, \ldots$ are analytic. System (10) is again transformed into

$$
\frac{\mathrm{d} r}{\mathrm{~d} \theta}=r \sum_{k=0}^{\infty} R_{k}(\theta) r^{k}
$$


where the function on the right-hand side of Equation (11) is convergent in the range $\theta \in[-4 \pi, 4 \pi],|r|<r_{0}$ ( $r_{0}$ is certain positive constant) and

$$
R_{k}(\theta+\pi)=(-1)^{k} R_{k}(\theta), k=0,1,2, \ldots
$$

For sufficiently small $h$, let

$$
\Delta(h)=r(2 \pi, h)-h, \quad r=r(\theta, h)=\sum_{m=1}^{\infty} v_{m}(\theta) h^{m}
$$

be the Poincaré succession function and the solution of Equation (11) satisfying the initial-value condition $\left.r\right|_{\theta=0}=h$. Moreover, for (13) we have

$$
v_{1}(\theta)=1, \quad v_{m}(0)=0, \quad m=2,3, \ldots
$$

Definition 2.2. For the succession function in (13), if $\nu_{2}(2 \pi)=\nu_{3}(2 \pi)=\cdots=\nu_{2 k}(2 \pi)$ $=0$ and $v_{2 k+1}(2 \pi) \neq 0$, then the origin is called the fine focus or weak focus of order $k$, and the quantity of $v_{2 k+1}(2 \pi)$ is called the $k$ th focal value at the origin on center manifold of system (8) or (2), $k=1,2, \ldots$.

Remark 1. For the coefficients of the form solution in (13), we have the following property $[13,15]$ : for every positive integer $m=1,2, \ldots$, there exists expression of the relation

$$
v_{2 m}(2 \pi)=\frac{1}{1+v_{1}(\pi)} \sum_{k=1}^{m-1} \xi_{m}^{(k)} v_{2 k+1}(2 \pi)
$$

where every $\xi_{m}^{(k)}$ is a polynomial of $v_{1}(\pi), v_{2}(\pi), \ldots, v_{2 m}(\pi)$ and $v_{1}(2 \pi), v_{2}(2 \pi), \ldots, v_{2 m}$ $(2 \pi)$ with rational coefficients. Particularly, if for each $1 \leq k \leq m-1, v_{2 k+1}(2 \pi)=0$ holds, we can get $v_{2}(2 \pi)=v_{4}(2 \pi)=\cdots=v_{2 m}(2 \pi)=0$.

\subsection{The singular point quantities on center manifold}

Here, we recall the definition of the singular point quantities on center manifold. By means of transformation

$$
z=x+y \mathbf{i}, \quad w=x-y \mathbf{i}, \quad u=u, \quad T=\mathbf{i} t, \quad \mathbf{i}=\sqrt{-1},
$$

system (8) is also transformed into the following complex system

$$
\left\{\begin{array}{l}
\frac{\mathrm{d} z}{\mathrm{~d} T}=z+\sum_{k+j+l=2}^{\infty} a_{k j} z^{k} w^{j} u^{l}=Z(z, w, u), \\
\frac{\mathrm{d} w}{\mathrm{~d} T}=-w-\sum_{k+j+l=2}^{\infty} b_{k j l} w^{k} z^{j} u^{l}=-W(z, w, u), \\
\frac{\mathrm{d} u}{\mathrm{~d} T}=\mathbf{i} d_{0} u+\sum_{k+j+l=2}^{\infty} \tilde{d}_{k j} z^{k} w^{j} u^{l}=\tilde{U}(z, w, u)
\end{array}\right.
$$

where z, $w, T, a_{k j l}, b_{k j l}, \tilde{d}_{k j l} \in \mathbb{C}(k, j, l \in \mathbb{N})$, the systems (8) and (17) are called concomitant. If no confusion arises, $\tilde{d}_{k j,}, \tilde{U}$ are still written as $d_{k j l}$ and $U$.

Lemma 2.1 (see [9]). For system (17), using the program of term by term calculations, we can determine a formal power series:

$$
F(z, w, u)=z w+\sum_{\alpha+\beta+\gamma=3}^{\infty} c_{\alpha \beta \gamma} z^{\alpha} w^{\beta} u^{\gamma}
$$


such that

$$
\frac{\mathrm{d} F}{\mathrm{~d} T}=\frac{\partial F}{\partial z} Z-\frac{\partial F}{\partial y} W+\frac{\partial F}{\partial u} U=\sum_{m=1}^{\infty} \mu_{m}(z w)^{m+1}
$$

where $c_{110}=1, c_{101}=c_{011}=c_{200}=c_{020}=0, c_{k k 0}=0, k=2,3, \ldots$. And $\mu_{m}$ is called the mth singular point quantity at the origin on center manifold of system (17) or (8) or (2).

Lemma 2.2 (see [9]). For the mth singular point quantity and the mth focal value at the origin on center manifold of system (8), i.e. $\mu_{m}$ and $v_{2 m+1}, m=1,2, \ldots$, we have the following relation:

$$
v_{2 m+1}(2 \pi)=\mathbf{i} \pi \mu_{m}+\mathbf{i} \pi \sum_{k=1}^{m-1} \xi_{m}^{(k)} \mu_{k}
$$

where $\xi_{m}^{(k)}(k=1,2, \ldots, m-1)$ are polynomial functions of coefficients of system (17). Usually it is called algebraic equivalence and written as $\nu_{2 m+1} \sim \mathbf{i} \pi \mu_{m}$.

\section{The conclusions and proofs}

\subsection{The equivalence for singular point quantities on center manifold}

In this subsection, we give firstly the results about the equivalence. Then the key theorem, i.e. the following Theorem 3.1 will be proved in next subsection.

Theorem 3.1. For the mth Liapunov constant of the origin for system (2) and the mth focal value at the origin for system (8), i.e. $V_{2 m}$ and $v_{2 m+1}$, for every positive integer $m$, we have the following relation:

$$
V_{2 m}=\sigma_{m} v_{2 m+1}(2 \pi)+\sum_{k=1}^{m-1} \xi_{m}^{(k)} v_{2 k+1}(2 \pi)
$$

where $\xi_{m}^{(k)}(k=1,2, \ldots, m-1)$ are polynomial functions of coefficients of system (8) and

$$
\boldsymbol{\sigma}_{m}=2 \kappa \ell \omega^{3}\left(\boldsymbol{v}^{2}+\boldsymbol{v}^{2}\right)\left(\int_{0}^{2 \pi}[\phi(\theta)]^{m+1} \mathrm{~d} \theta\right)^{-1},
$$

with

$$
\phi(\theta)= \begin{cases}x_{1 \theta}^{2}, & \text { if } \Phi=x_{1}^{2} \\ x_{2 \theta}^{2}, & \text { if } \Phi=x_{2}^{2} \\ x_{1 \theta}^{2}+x_{2 \theta}^{2}, & \text { if } \Phi=x_{1}^{2}+x_{2}^{2} \\ \left(x_{1 \theta}+x_{2 \theta}\right)^{2}, & \text { if } \Phi=\left(x_{1}+x_{2}\right)^{2}\end{cases}
$$

where

$$
x_{1 \theta}=\left[\left(\ell_{1} v+\omega v\right) \cos (\theta)+\left(\ell_{1} v-\omega v\right) \sin (\theta)\right] / \ell_{,} \quad x_{2 \theta}=v \cos (\theta)+v \sin (\theta)
$$

and $\boldsymbol{v}, \boldsymbol{v}$ are the two constants given by the real transformation matrix $P$ in (7) with $v^{2}+v^{2} \neq 0$. Then, we also call the relation algebraic equivalence and write as $V_{2 m} \sim$ $\sigma_{m} v_{2 m+1}(2 \pi), m=1,2, \ldots$.

How to determine the above $\boldsymbol{v}, \boldsymbol{v}$ is shown in the next elementary lemma.

Lemma 3.2. The nondegenerate real transformation matrix in (7) possesses necessarily the following generic form: 


$$
P=\left(\begin{array}{ccc}
\frac{v \ell_{1}+v \omega}{\ell} & \frac{v \ell_{1}-v \omega}{\ell} & 0 \\
v & v & 0 \\
0 & 0 & \zeta
\end{array}\right)
$$

where $\boldsymbol{\varsigma}, \boldsymbol{v}, \boldsymbol{v}$ are three real numbers such that $\boldsymbol{\varsigma}\left(\boldsymbol{v}^{2}+\boldsymbol{v}^{2}\right) \neq 0$ holds.

Proof. Considering that the coefficient matrix $A$ in (7) has a pair of purely imaginary eigenvalues $\mathbf{i} \omega,-\mathbf{i} \omega$ and one real eigenvalue $-d$, one can select freely their eigenvectors respectively, for example

$$
\eta_{1}=\left(\frac{\ell_{1}+\mathbf{i} \omega}{\ell}, 1,0\right)^{\prime}, \quad \eta_{2}=\left(\frac{\ell_{1}-\mathbf{i} \omega}{\ell}, 1,0\right)^{\prime}, \eta_{3}=(0,0,1)^{\prime} .
$$

Thus, there must exist only a generic transformation matrix $T_{1}=k_{1} \eta_{1}+k_{2} \eta_{2}+k_{3} \eta_{3}$ with the following form

$$
T_{1}=\left(\begin{array}{ccc}
\frac{k_{1}\left(\ell_{1}+i \omega\right)}{\ell} & \frac{k_{2}\left(\ell_{1}-i \omega\right)}{\ell} & 0 \\
k_{1} & k_{2} & 0 \\
0 & 0 & k_{3}
\end{array}\right)
$$

such that

$$
T_{1}^{-1} A T_{1}=\left(\begin{array}{ccc}
\mathbf{i} \omega & 0 & 0 \\
0 & -\mathbf{i} \omega & 0 \\
0 & 0 & -d
\end{array}\right) \triangleq J
$$

where $k_{1}, k_{2}$ and $k_{3}$ are three arbitrary non-zero constants.

Similarly, we obtain also that for the matrix $B$ in (7), there must exist only a generic transformation matrix $T_{2}$ with the following form

$$
T_{2}=\left(\begin{array}{ccc}
\mathbf{i} j_{1} & -\mathbf{i} j_{2} & 0 \\
j_{1} & j_{2} & 0 \\
0 & 0 & j_{3}
\end{array}\right)
$$

such that

$$
T_{2}^{-1} B T_{2}=\left(\begin{array}{ccc}
\mathbf{i} \omega & 0 & 0 \\
0 & -\mathbf{i} \omega & 0 \\
0 & 0 & -d
\end{array}\right)=J
$$

where $j_{1}, j_{2}$ and $j_{3}$ are also three arbitrary non-zero constants.

Then from (26) and (27), there must exist only a generic transformation matrix $P$ as follows

$$
P=T_{1} T_{2}^{-1}=\left(\begin{array}{ccc}
\frac{\ell_{1}\left(K_{2}-K_{1}\right) \mathbf{i}+\left(K_{2}+K_{1}\right) \omega}{2 \ell} & \frac{\left(K_{2}+K_{1}\right) \ell_{1}-\left(K_{2}-K_{1}\right) \omega \mathbf{i}}{2 \ell} & 0 \\
\frac{\left(K_{2}-K_{1}\right) \mathbf{i}}{2} & \frac{K_{2}+K_{1}}{2} & 0 \\
0 & 0 & \zeta
\end{array}\right)
$$

such that $P^{-1} A P=B$ in (7) holds, and where $K_{1}=k_{1} / j_{1}, K_{2}=k_{2} / j_{2}$ and $s=k_{3} / j_{3}$ are also arbitrary non-zero numbers because of the property of $k_{1}, k_{2}, k_{3}, j_{1}, j_{2}$ and $j_{3}$. Furthermore in order to guarantee that the transformation in (7) is real and nondegenerate, form (28), there is no other choice, only we can let $K_{1}$ and $K_{2}$ conjugate, i.e.

$$
K_{1}=v+\mathrm{i} v, K_{2}=v-\mathrm{i} v, v, v, \in \mathbb{R},
$$


at the same time, $\boldsymbol{c}, \boldsymbol{v}$ and $\boldsymbol{v}$ are three real numbers such that $|P| \neq 0$ holds, thus we obtain the generic form of the transformation matrix $P$.

Remark 2. From the expression $\sigma_{m}, m=1,2, \ldots$ in (22), one should notice that once the coefficient matrix $A$ and transformation matrix $P$ are given explicitly, then $\sigma_{m}$ is never an arbitrary and undefined value. In particular, when $\lambda=-\lambda_{2}=\omega, \lambda_{1}=0$ in system (2), i.e. $A=B$ in (7), we can get the identity matrix $E$ as the simplest transformation matrix $P$, namely $v=1, v=0$, if we choose

$$
\kappa=\frac{1}{2} \omega^{-4} \quad \text { and } \quad \Phi=x_{1}^{2}+x_{2}^{2}
$$

in (6), then at this time every $\sigma_{m}=\frac{1}{2 \pi}$.

Furthermore, from Lemma 2.2 and Theorem 3.1, we have

Theorem 3.3. For the mth Liapunov constant of the origin for system (2) and the mth singular point quantity of the origin for system (8) or (17), i.e. $V_{2 m}$ and $\mu_{m}, m=1,2, \ldots$, there exists the following relation:

$$
V_{2 m}=\mathbf{i} \pi \boldsymbol{\sigma}_{m} \mu_{m}+\mathbf{i} \pi \boldsymbol{\sigma}_{m} \sum_{k=1}^{m-1} \xi_{m}^{(k)} \mu_{k}
$$

where $\sigma_{m}$ has been given in (22) of Theorem 3.1 and $\xi_{m}^{(k)}(k=1,2, \ldots, m-1)$ are polynomial functions of coefficients of system (17). Similarly we also call it algebraic equivalence and write as $V_{2 m} \sim \mathbf{i} \pi \boldsymbol{\sigma}_{m} \mu_{m}$.

Remark 3. From (20) in Lemma 2.2 and (29) in Theorem 3.3, we get that:

$$
V_{2}=\sigma_{1} v_{3}(2 \pi)=\mathbf{i} \pi \sigma_{1} \mu_{1}
$$

and if for all $k=1,2, \ldots, m-1, V_{2 k}=0$ or $v_{2 k+1}=0$ or $\mu_{k}=0$ holds, then we have

$$
V_{2 m}=\sigma_{m} v_{2 m+1}=\mathbf{i} \pi \boldsymbol{\sigma}_{m} \mu_{m}, \quad m=2,3, \ldots
$$

Thus the stability of the origin for the systems (1) or (2) can be figured out directly by calculating the singular point quantities of the origin for system (17).

\subsection{Proof of theorem 3.1}

Firstly, from Lemma 3.2 and the nondegenerate transformation: $\left(x_{1}, x_{2}, x_{3}\right)^{\prime}=P(x, y, u)^{\prime}$ in (7), we obtain

$$
\left(x_{1}, x_{2}, x_{3}\right)=\left(\frac{\boldsymbol{v} \ell_{1}+v \omega}{\ell} x+\frac{\boldsymbol{v} \ell_{1}-\boldsymbol{v} \omega}{\ell} y, \boldsymbol{v} x+\boldsymbol{v} y, \zeta u\right),
$$

and more under the polar coordinates $x=r \cos \theta, y=r \sin \theta$, we have

$$
\begin{aligned}
& x_{1}=\frac{r}{\ell}\left[\left(\boldsymbol{v} \ell_{1}+\boldsymbol{v} \omega\right) \cos (\theta)+\left(\boldsymbol{v} \ell_{1}-\boldsymbol{v} \omega\right) \sin (\theta)\right]=r x_{1 \theta}, \\
& x_{2}=r[\boldsymbol{v} \cos (\theta)+\boldsymbol{v} \sin (\theta)]=r x_{2 \theta} .
\end{aligned}
$$

Substituting (32) into the Liapunov function (5), then we denote

$$
H\left(x_{1}, x_{2}\right)=H[x, y]=\sum_{k=2}^{\infty} H_{k}[x, y]
$$

where $H_{k}[x, y]$ is a homogeneous polynomial in $x, y$ of degree $k$ and

$$
H_{2}[x, y]=-\frac{\kappa}{\ell}\left(v^{2}+v^{2}\right)\left(\ell_{1}^{2}+\ell \ell_{2}\right)\left(x^{2}+y^{2}\right)
$$


with $H_{2}[1,0]=\frac{\kappa}{\ell}\left(\boldsymbol{v}^{2}+\boldsymbol{v}^{2}\right) \omega^{2}$. And more from the expressions (13) and (34), we have

$$
\begin{aligned}
H[x, y] & =H[r(\theta, h) \cos (\theta), r(\theta, h) \sin (\theta)]=\sum_{k=2}^{\infty} H_{k}[\cos (\theta), \sin (\theta)] r^{k}(\theta, h) \\
& =\hat{H}(\theta, h) .
\end{aligned}
$$

Next, we investigate $\Delta \hat{H}=\hat{H}(2 \pi, h)-\hat{H}(0, h)$ based on the Equation 6 .

On the one hand, from (14), we denote

$$
\begin{aligned}
\hat{H}(2 \pi, h)-\hat{H}(0, h) & =\sum_{k=2}^{\infty} H_{k}[1,0]\left[r^{k}(2 \pi, h)-r^{k}(0, h)\right] \\
& =\sum_{k=2}^{\infty} H_{k}[1,0]\left[r^{k}(2 \pi, h)-h^{k}\right] \\
& =\Delta \hat{H}_{1}
\end{aligned}
$$

where

$$
\begin{aligned}
r^{k}(2 \pi, h)-h^{k} & =(r(2 \pi, h)-h)\left(r^{k-1}(2 \pi, h)+r^{k-2}(2 \pi, h) h+\cdots+h^{k-1}\right) \\
& =(r(2 \pi, h)-h)\left(k h^{k-1}+o\left(h^{k-1}\right)\right),
\end{aligned}
$$

and from the expression (13), one can get

$$
\begin{aligned}
\Delta \hat{H}_{1} & =(r(2 \pi, h)-h) \sum_{k=2}^{\infty} H_{k}[1,0]\left(k h^{k-1}+o\left(h^{k-1}\right)\right) \\
& =(r(2 \pi, h)-h)\left[\frac{2}{\ell} \kappa\left(\boldsymbol{v}^{2}+\boldsymbol{v}^{2}\right) \omega^{2} h+o(h)\right] \\
& =\frac{2}{\ell} \kappa\left(\boldsymbol{v}^{2}+\boldsymbol{v}^{2}\right) \omega^{2} \sum_{m=2}^{\infty} v_{m}(2 \pi) h^{m+1}(1+o(1)) .
\end{aligned}
$$

On the other hand, we denote

$$
\hat{H}(2 \pi, h)-\hat{H}(0, h)=\int_{0}^{2 \pi} \frac{\mathrm{d} \hat{H}}{\mathrm{~d} \theta} \mathrm{d} \theta=\int_{0}^{2 \pi} \frac{\mathrm{d} \hat{H}}{\mathrm{~d} \tau} \frac{\mathrm{d} \tau}{\mathrm{d} \theta} \mathrm{d} \theta=\frac{1}{\omega} \int_{0}^{2 \pi} \frac{\mathrm{d} \hat{H}}{\mathrm{~d} \tau} \frac{\mathrm{d} t}{\mathrm{~d} \theta} \mathrm{d} \theta=\Delta \hat{H}_{2} .
$$

From system (10) and the expression (13), we can get

$$
\frac{\mathrm{d} t}{\mathrm{~d} \theta}=\left[1+\sum_{k=2}^{\infty} r^{k-1} \psi_{k+1}(\theta)\right]^{-1}=1+c_{1}(\theta) h+c_{2}(\theta) h^{2}+\cdots=1+o(1)
$$

where $\psi_{k+1}(\theta), c_{k-1}(\theta)(k=2,3, \ldots)$ are analytic. At the same time, putting (33) in the right side of (6), we have

$$
\frac{\mathrm{d} \hat{H}}{\mathrm{~d} \tau}=\sum_{m=1}^{\infty} V_{2 m}\left[\phi^{\frac{1}{2}}(\theta) r(\theta, h)\right]^{2 m+2}=\sum_{m=1}^{\infty} V_{2 m} h^{2 m+2}[\phi(\theta)]^{m+1}(1+o(1)),
$$

thus

$$
\Delta \hat{H}_{2}=\frac{1}{\omega} \sum_{m=1}^{\infty}\left[V_{2 m} h^{2 m+2}\left(\int_{0}^{2 \pi}[\phi(\theta)]^{m+1} \mathrm{~d} \theta+o(1)\right)\right] .
$$


According to (39), (43) and applying (15) in Remark 1 and mathematical induction to $m$, we complete the proof.

\section{Singular point quantities for the Lü system}

In this section, we investigate the singular point quantities of the equilibrium point of the Lü system which bridges the gap between the Lorenz and Chen attractors [7], and takes the following form

$$
\left\{\begin{array}{l}
\dot{x}_{1}=a\left(x_{2}-x_{1}\right) \\
\dot{x}_{2}=c x_{2}-x_{1} x_{3} \\
\dot{x}_{3}=x_{1} x_{2}-b x_{3}
\end{array}\right.
$$

where $a>0, b>0, c>0$. Obviously, system (44) has three equilibrium points: $O(0,0,0)$, $O_{1}(\sqrt{b c}, \sqrt{b c}, c)$ and $O_{2}(-\sqrt{b c},-\sqrt{b c}, c)$. Because the Jacobian matrix at the origin $O$ has no purely imaginary eigenvalues, it is unnecessary to consider its singular point quantities. And more the equations in (44) are invariant under the transformation:

$$
\left(x_{1}, x_{2}, x_{3}\right) \mapsto\left(-x_{1},-x_{2}, x_{3}\right),
$$

which means that system (44) is symmetrical. Therefore, we only need to consider $O_{1}$.

The Jacobian matrix of system (44) at $O_{1}$ is

$$
A_{o}=\left(\begin{array}{ccc}
-a & a & 0 \\
-c & c & -\sqrt{b c} \\
\sqrt{b c} & \sqrt{b c} & -b
\end{array}\right)
$$

with the characteristic equation: $\lambda^{3}+(a+b-c) \lambda+a b \lambda+2 a b c=0$. To guarantee that $A_{o}$ has a pair of purely imaginary eigenvalues $\pm \mathbf{i} \omega(\omega>0)$ and one negative real eigenvalue $\lambda_{0}$, we let its characteristic equation take the form

$$
\left(\lambda^{2}+\omega^{2}\right)\left(\lambda-\lambda_{0}\right)=0 .
$$

Thus we obtain the critical condition of Hopf bifurcation at $O_{1}: c=(a+b) / 3$, then

$$
\omega=\sqrt{b c}, \quad \lambda_{0}=-2 c
$$

namely $b=\omega^{2} / a, c=\left(a^{2}+\omega^{2}\right) /(3 a)$. By the translation: $\left(x_{1}, x_{2}, x_{3}\right) \rightarrow\left(x_{1}+\omega, x_{2}+\right.$ $\left.\omega, x_{3}+c\right)$, we make the equilibrium $O_{1}$ become the origin and change system (44) into

$$
\dot{\mathbf{x}}=A_{o}\left(\begin{array}{l}
x_{1}+\omega \\
x_{2}+\omega \\
x_{3}+c
\end{array}\right)+\left(\begin{array}{c}
0 \\
-\left(x_{1}+\omega\right)\left(x_{3}+c\right) \\
\left(x_{1}+\omega\right)\left(x_{2}+\omega\right)
\end{array}\right) .
$$

Under the conditions (46), one can find a nondegenerate matrix

$$
P_{o}=\left(\begin{array}{ccc}
\frac{\sqrt{3 a}\left(2 a^{2}-\omega^{2}\right)}{d_{1} \sqrt{d_{0}}} & \frac{3 \sqrt{3} a^{2} \omega}{d_{1} \sqrt{d_{0}}} & -\frac{\sqrt{3} a^{2}}{2 \omega \omega d_{0}} \\
\frac{2 a \sqrt{3 d_{0}}}{d_{1}} & \frac{\omega \sqrt{3 d_{0}}}{d_{1}} & \frac{2 \omega^{2}-a^{2}}{2 \omega \sqrt{3 d_{0}}} \\
0 & 1 & 1
\end{array}\right)
$$

such that

$$
P_{o}^{-1} A_{o} P_{o}=\left(\begin{array}{ccc}
0 & -\omega & 0 \\
\omega & 0 & 0 \\
0 & 0 & -2 d_{0} /(3 a)
\end{array}\right)
$$


where $d_{0}=a^{2}+\omega^{2}, d_{1}=4 a^{2}+\omega^{2}$.

Then we can use the nondegenerate transformation $\mathbf{x}=P_{o} \mathbf{y}$ and the time re-scaling: $t \rightarrow t / \omega$ to make the system (47) become the following form:

$$
\begin{aligned}
\dot{y} & =\frac{1}{\omega}\left[P_{o}^{-1} * \operatorname{diag}\left(P_{o} y+E\right) A_{o} P_{o} y\right] \\
& =\frac{1}{\omega}\left[P_{o}^{-1} A_{o} P_{o} y+P_{o}^{-1} * \operatorname{diag}\left(P_{o} y\right) A_{o} P_{o} y\right]
\end{aligned}
$$

where $\mathbf{y}=\left(y_{1}, y_{2}, y_{3}\right)$, and $E$ is the $3 \times 3$ identity matrix.

Now we put $y_{1}=(z+w) / 2, y_{2}=(z-w) \mathbf{i} / 2, y_{3}=u, t=-T \mathbf{i}$ in system (49) and obtain the following same form as the complex system (17):

$$
\left\{\begin{array}{l}
\frac{\mathrm{d} z}{\mathrm{~d} T}=z+a_{101} u z+a_{011} u w+a_{110} z w+a_{200} z^{2}+a_{020} w^{2}+a_{002} u^{2}=Z \\
\frac{\mathrm{d} w}{\mathrm{~d} T}=-\left(w+b_{011} u z+b_{101} u w+b_{110} z w+b_{020} z^{2}+b_{200} w^{2}+b_{002} u^{2}\right)=-W \\
\frac{\mathrm{d} u}{\mathrm{~d} T}=d_{001} u+d_{101} u z+d_{011} u w+d_{110} z w+d_{200} z^{2}+d_{020} w^{2}+d_{002} u^{2}=U
\end{array}\right.
$$

where $u \in \mathbb{R}, z, w, T \in \mathbb{C}$, and

$$
\begin{aligned}
& a_{200}=\frac{3 a\left(7 a^{2}+4 \omega^{2}\right)}{2 d_{1} d_{2}}+\frac{9 a^{4}}{2 d_{1} d_{2} \omega} \mathbf{i} \\
& a_{020}=-\frac{3 a\left(16 a^{6}-93 a^{2} \omega^{4}+4 \omega^{6}\right)}{2 d_{1}^{3} d_{2}}+\frac{3 a^{2}\left(32 \omega^{6}-16 a^{6}-72 a^{4} \omega^{2}-105 a^{2} \omega^{4}\right)}{2 d_{1}^{3} d_{2} \omega} \mathbf{i}, \\
& a_{002}=-\frac{3 a^{3}\left(8 a^{4}+19 a^{2} \omega^{2}+20 \omega^{4}\right)}{8 d_{0} d_{1} d_{2} \omega^{2}}-\frac{a^{2}\left(4 a^{6}-21 a^{4} \omega^{2}-36 a^{2} \omega^{4}+16 \omega^{6}\right)}{8 d_{0} d_{1} d_{2} \omega^{3}} \mathbf{i}, \\
& a_{101}=-\frac{d_{0} a\left(5 a^{2}-4 \omega^{2}\right)}{d_{1} d_{2} \omega^{2}}+\frac{3 a^{2}\left(5 a^{2}-4 \omega^{2}\right)}{2 d_{1} d_{2} \omega} \mathbf{i}, \\
& a_{011}=-\frac{a\left(a^{2}-2 \omega^{2}\right)\left(8 a^{6}+30 a^{4} \omega^{2}+45 a^{2} \omega^{4}-4 \omega^{6}\right)}{2 d_{0} d_{1}^{2} d_{2} \omega^{2}}+\frac{3 a^{2}\left(a^{2}-2 \omega^{2}\right)\left(4 a^{4}+5 a^{2} \omega^{2}-8 \omega^{4}\right)}{2 d_{0} d_{1}^{2} d_{2} \omega} \mathbf{i}, \\
& a_{110}=\frac{18 a^{3}\left(2 a^{2}+5 \omega^{2}\right)}{d_{1}^{2} d_{2}}+\frac{3 a^{2}\left(8 \omega^{4}-4 a^{4}-23 a^{2} \omega^{2}\right)}{d_{1}^{2} d_{2} \omega} \mathbf{i}, \\
& b_{k j l}=\bar{a}_{k j l}(k j l=200,020,002,101,011,110), \\
& d_{200}=-\frac{6 a\left(16 a^{6}-36 a^{4} \omega^{2}-21 a^{2} \omega^{4}+4 \omega^{6}\right)}{d_{1}^{3} d_{2}}+\frac{54 a^{2} \omega\left(4 a^{4}-a^{2} \omega^{2}-2 \omega^{4}\right)}{d_{1}^{3} d_{2}} \mathbf{i}, \\
& d_{020}=\frac{6 a\left(16 a^{6}-36 a^{4} \omega^{2}-21 a^{2} \omega^{4}+4 \omega^{6}\right)}{d_{1}^{3} d_{2}}+\frac{54 a^{2} \omega\left(4 a^{4}-a^{2} \omega^{2}-2 \omega^{4}\right)}{d_{1}^{3} d_{2}} \mathbf{i}, \\
& d_{002}=-\frac{3 a^{2}\left(5 a^{2}-4 \omega^{2}\right)}{2 d_{1} d_{2} \omega} \mathbf{i}, \\
& d_{101}=-\frac{3 a\left(16 a^{4}-7 a^{2} \omega^{2}+4 \omega^{4}\right)}{d_{1}^{2} d_{2}}-\frac{12 a^{2}\left(2 a^{2}-\omega^{2}\right)\left(a^{2}-2 \omega^{2}\right)}{d_{1}^{2} d_{2} \omega} \mathbf{i}, \\
& d_{011}=\frac{3 a\left(16 a^{4}-7 a^{2} \omega^{2}+4 \omega^{4}\right)}{d_{1}^{2} d_{2}}-\frac{12 a^{2}\left(2 a^{2}-\omega^{2}\right)\left(a^{2}-2 \omega^{2}\right)}{d_{1}^{2} d_{2} \omega} \mathbf{i}, \\
& d_{110}=\frac{36 a^{2} \omega\left(a^{2}-2 \omega^{2}\right)}{d_{1}^{2} d_{2}} \mathbf{i}, \\
& d_{001}=\frac{2 d_{1}}{3 a \omega} \mathbf{i}
\end{aligned}
$$

where $d_{2}=a^{2}+4 \omega^{2}$, and $\bar{a}_{k j l}$ denotes the conjugate complex number of $a_{k j l}$.

According to Lemma 2.1, we obtain the recursive formulas $c_{\alpha \beta \gamma}$ and $\mu_{m}$ in Appendix. By applying the formulas in the Mathematica symbolic computation system, we figure out easily the first twenty singular point quantities of the origin of system (50):

$$
\begin{aligned}
& \mu_{1}=-243 \mathbf{i} a^{5}\left(2 a^{2}-\omega^{2}\right)\left(a^{2}-5 \omega^{2}\right) \omega /\left(d_{0} d_{1}^{2} d_{2} d_{3}\right), \\
& \mu_{2}=-891849714087780 \mathbf{i} a^{7}\left(2 a^{2}-\omega^{2}\right) \omega^{21} /\left(d_{0}^{3} d_{1}^{5} d_{2}^{3} d_{3}^{2} d_{4}\right), \\
& \mu_{3}=\mu_{4}=\cdots=\mu_{20}=0
\end{aligned}
$$

where $d_{3}=a^{4}+11 a^{2} \omega^{2}+\omega^{4}, d_{4}=4 a^{4}+89 a^{2} \omega^{2}+4 \omega^{4}$, and in the above expression of each $\mu_{k}, k=2,3, \ldots$, we have already let $\mu_{1}=\cdots=\mu_{k-1}=0$. 
From the remark 3 and the singular point quantities (51), if we let $P_{o}^{-1} A_{o} P_{o}$ in (48) become $A$ in (7), and considering the particular case in the Remark 2, then we have

Theorem 4.1. For the flow on center manifold of the system (44), the first two focal values and Liapunov constants of the origin are as follow

$$
\begin{aligned}
& \text { (i) } v_{3}=\mathbf{i} \pi \mu_{1}, \quad v_{5}=\mathbf{i} \pi \mu_{2} \\
& \text { (ii) } V_{2}=\frac{1}{2} \mathbf{i} \mu_{1}, \quad V_{4}=\frac{1}{2} \mathbf{i} \mu_{2}
\end{aligned}
$$

where the expression of $v_{5}$ is obtained under the condition of $v_{3}=0$, and $V_{4}$ is obtained under the condition of $V_{2}=0$.

Remark 4. Considering Hopf bifurcation at the two symmetrical equilibria $O_{1}$ and $O_{2}$, form the Theorem 4.1, we conclude that the Lü system (44) at least 4 small limit cycles, which will be proved rigorously in a following paper.

\section{Appendix}

$$
\begin{aligned}
c_{\alpha \beta \gamma}= & \frac{1}{\beta-\alpha-\gamma d_{0}}\left[d_{200}(1+\gamma) c_{\alpha-2, \beta, \gamma+1}-b_{020}(1+\beta) c_{\alpha-2, \beta+1, \gamma}+d_{110}(1+\gamma) c_{\alpha-1, \beta-1, \gamma+1}-\right. \\
& \left(a_{200}+b_{110} \beta-a_{200} \alpha-d_{101} \gamma\right) c_{\alpha-1, \beta, \gamma}-b_{011}(1+\beta) c_{\alpha-1, \beta+1, \gamma-1}+ \\
& d_{020}(\gamma+1) c_{\alpha, \beta-2, \gamma+1}+\left(b_{200}-b_{200} \beta+a_{110} \alpha+d_{011} \gamma\right) c_{\alpha, \beta-1, \gamma}-\left(d_{002}+b_{101} \beta-\right. \\
& \left.a_{101} \alpha-d_{002} \gamma\right) c_{\alpha, \beta, \gamma-1}-b_{002}(1+\beta) c_{\alpha, \beta+1, \gamma-2}+a_{020}(1+\alpha) c_{\alpha+1, \beta-2, \gamma}+ \\
& \left.a_{011}(1+\alpha) c_{\alpha+1, \beta-1, \gamma-1}+a_{002}(1+\alpha) c_{\alpha+1, \beta, \gamma-2}\right] \\
\mu_{m}= & d_{200} c_{m-2, m, 1}-b_{020}(1+m) c_{m-2, m+1,0}+d_{110} c_{m-1, m-1,1}-\left(a_{200}-a_{200} m+b_{110} m\right) \\
& c_{m-1, m, 0}+d_{020} c_{m, m-2,1}+\left(b_{200}+a_{110} m-b_{200} m\right) c_{m, m-1,0}+a_{020}(1+m) c_{m+1, m-2,0} .
\end{aligned}
$$

\section{Acknowledgements}

This work was supported by the National Natural Science Foundation of China (Project No. 10961011) and the Science Fund of Hubei Province Education Department in China (Project No. Q20091209).

\section{Author details}

${ }^{1}$ School of Information and Mathematics, Yangtze University, Jingzhou 434023, People's Republic of China ${ }^{2}$ School of Mathematics and Computing Science, Guilin University of Electronic Technology, Guilin 541004, People's Republic of China

\section{Authors' contributions}

QW completed the main study, carried out the results of this article and drafted the manuscript. WH checked the proofs and verified the calculation. All the authors read and approved the final manuscript.

\section{Competing interests}

The authors declare that they have no competing interests.

Received: 14 January 2012 Accepted: 7 June 2012 Published: 7 June 2012

\section{References}

1. Hofbauer, J, Sigmund, K: Evolutionary Games and Population Dynamics. Cambridge University Press, Cambridge (1998)

2. Zeeman, ML: Hopf bifurcations in competitive three-dimensional Lotka-Volterra systems. Dyn Stab Syst. 8, 189-217 (1993). doi:10.1080/02681119308806158

3. Sparrow, C: The Lorenz Equations: Bifcations, Chaos, and Strange Attractors. Springer, New York (1982)

4. Celikovský, S, Chen, G: On the generalized Lorenz canonical form. Chaos, Solitons Fractals. 26, 1271-1276 (2005). doi:10.1016/j.chaos.2005.02.040

5. Carr, J: Applications of Center Manifold Theory. Springer, New York (1981)

6. Hassard, B, Kazarinoff, N, Wan, Y: Theory and Application of Hopf Bifurcation. Cambridge University Press, Cambridge (1981)

7. Lu, Z, Luo, Y: Two limit cycles in three-dimensional Lotka-Volterra systems. Comput Math Appl. 44, 51-66 (2002). doi:10.1016/S0898-1221(02)00129-3

8. Kuznestov, YA: Elements of applied bifurcation theory. Springer, New York (1998)

9. Wang, Q, Liu, Y, Chen, H: Hopf bifurcation for a class of three-dimensional nonlinear dynamic systems. Bull des Sci Math. 134, 786-798 (2010). doi:10.1016/j.bulsci.2009.12.001 
10. Andronov, AA, Leontovich, EA, Gordon, II, Maier, AG: Theory of Bifurcation of Dynamic Systems on a Plane. Wiley, New York (1973)

11. Wang, D: Mechanical manipulation for a class of differential systems. J Symb Comput. 12, $233-254$ (1991). doi:10.1016/ S0747-7171(08)80127-7

12. Liu, Y, Li, J: Theory of values of singular point in complex autonomous differential system. Sci China Ser A. 33, 10-24 (1990)

13. Liu, Y: Theory of center-focus for a class of higher-degree critical points and infinite points. Sci China Ser A. 44, 37-48 (2001)

14. Chen, $\mathrm{H}, \mathrm{Liu}, \mathrm{Y}$ : Linear recursion formulas of quantities of singular point and applications. Appl Math Comput. 148, 163-171 (2004). doi:10.1016/50096-3003(02)00835-4

15. Liu, Y, Huang, W: A cubic system with twelve small amplitude limit cycles. Bull des Sci Math. 129, 83-98 (2005). doi:10.1016/j.bulsci.2004.05.004

doi:10.1186/1687-1847-2012-78

Cite this article as: Wang and Huang: The equivalence between singular point quantities and Liapunov constants on center manifold. Advances in Difference Equations 2012 2012:78.

\section{Submit your manuscript to a SpringerOpen ${ }^{\circ}$} journal and benefit from:

- Convenient online submission

- Rigorous peer review

- Immediate publication on acceptance

- Open access: articles freely available online

- High visibility within the field

- Retaining the copyright to your article

Submit your next manuscript at $\gg$ springeropen.com 